\title{
A parametric branch and bound approach to suboptimal explicit hybrid MPC
}

Daniel Axehill, Thomas Besselmann, Davide Martino Raimondo and Manfred Morari

\section{Linköping University Post Print}

\section{Tweet}

N.B.: When citing this work, cite the original article.

Original Publication:

Daniel Axehill, Thomas Besselmann, Davide Martino Raimondo and Manfred Morari, A parametric branch and bound approach to suboptimal explicit hybrid MPC, 2014, Automatica, (50), 1, 240-246.

http://dx.doi.org/10.1016/j.automatica.2013.10.004

Copyright: Elsevier / International Federation of Automatic Control (IFAC) http://www.ifac-control.org/

Postprint available at: Linköping University Electronic Press

http://urn.kb.se/resolve?urn=urn:nbn:se:liu:diva-105420 
Brief paper

\title{
A parametric branch and bound approach to suboptimal explicit hybrid MPC
}

\author{
Daniel Axehill ${ }^{\mathrm{a}, \mathrm{d}, 1}$, Thomas Besselmann ${ }^{\mathrm{b}, \mathrm{d}}$, Davide Martino Raimondo ${ }^{\mathrm{c}, \mathrm{d}}$, \\ Manfred Morari ${ }^{\mathrm{d}}$ \\ a Linköping University, Division of Automatic Control, 58183 Linköping, Sweden \\ ${ }^{\mathrm{b}}$ ABB Corporate Research, Automation and Control, 5405 Baden-Dättwil, Switzerland \\ c University of Pavia, Dipartimento di Ingegneria Industriale e dell'Informazione, 27100 Pavia, Italy \\ d ETH Zurich, Automatic Control Laboratory, 8092 Zurich, Switzerland
}

\section{A R T I C L E I N F O}

\section{Article history:}

Received 10 February 2012

Received in revised form

11 June 2013

Accepted 24 September 2013

Available online 1 November 2013

\section{Keywords:}

Suboptimal

Explicit MPC

Hybrid systems

Branch and bound

Stability

\begin{abstract}
A B S T R A C T
In this article we present a parametric branch and bound algorithm for computation of optimal and suboptimal solutions to parametric mixed-integer quadratic programs and parametric mixed-integer linear programs. The algorithm returns an optimal or suboptimal parametric solution with the level of suboptimality requested by the user. An interesting application of the proposed parametric branch and bound procedure is suboptimal explicit MPC for hybrid systems, where the introduced user-defined suboptimality tolerance reduces the storage requirements and the online computational effort, or even enables the computation of a suboptimal MPC controller in cases where the computation of the optimal MPC controller would be intractable. Moreover, stability of the system in closed loop with the suboptimal controller can be guaranteed a priori.
\end{abstract}

(C) 2013 Elsevier Ltd. All rights reserved.

\section{Introduction}

Many problems in control require the solution of mixed-integer programs which involve continuous and discrete optimization variables. One example is hybrid systems, where continuous and discrete dynamics interact. In this paper, we consider mixed logical dynamical (MLD) systems. The MLD system class is a common modeling class for hybrid phenomena, which, under some technical assumptions, is equivalent to many other hybrid system classes, Heemels, de Schutter, and Bemporad (2001).

A common technique to control hybrid systems is Model Predictive Control (MPC), Camacho and Bordons (2004). MPC is a

\footnotetext{
is This research has been partially supported by the Swiss National Science Foundation under grant 200021-122072, by the European Commission under the project Feednetback FP7-ICT-223866 (www.feednetback.eu), by the Swedish Research Council under contract Nr. B0598501, and by CENIIT at LiU. The material in this paper was partially presented at the 18th IFAC World Congress, August 28-September 2, 2011, Milan, Italy. This paper was recommended for publication in revised form by Associate Editor Martin Guay under the direction of Editor Frank Allgöwer.

E-mail addresses: daniel@isy.liu.se, axehill@control.ee.ethz.ch (D. Axehill), besselmann@control.ee.ethz.ch (T. Besselmann),

davide.raimondo@control.ee.ethz.ch (D.M. Raimondo), morari@control.ee.ethz.ch (M. Morari)

1 Tel.: +46 13 284042; fax: +4613139282.
}

flexible control methodology which can take hybrid phenomena as well as hard constraints on states and inputs into account. In MPC, optimization problems are solved repeatedly at each sampling instance, in what is called the receding horizon control strategy. The major drawback of MPC is the computational effort. In order to mitigate this drawback a technique based on parametric programming called Explicit MPC, has been developed, Bank, Guddat, Klatte, Kummer, and Tammer (1982) and Bemporad, Morari, Dua, and Pistikopoulos (2002). Parametric programming allows one to solve the optimal control problems not only for a single, but for a set of states, and thus to shift the computational effort from online to offline. Even though this potentially allows for fast online applications, the complexity of the solution that is sought for might make the process intractable already at the point of offline computation of the explicit solution. This situation motivated the development of a variety of different approximate explicit MPC schemes, see, e.g., Summers, Jones, Lygeros, and Morari (2011).

In the case of hybrid systems and a quadratic cost function, the computation of explicit MPC control laws boils down to solving a parametric mixed-integer quadratic program. Several solution strategies have been proposed, based on the solution of mixedinteger nonlinear programs (MINLP), Dua, Bozinis, and Pistikopoulos (2002), the enumeration of all switching sequences, Kvasnica, Grieder, Baotic, and Morari (2004), or dynamic programming, Borrelli (2003) and Baotic (2005). 
In this article, the focus is on parametric mixed-integer quadratic problems. We introduce a parametric branch and bound procedure to search for suboptimal solutions with a guaranteed bound on the suboptimality. The proposed procedure does not require knowledge about the optimal solution. In the MPC framework this allows the user to enforce guarantees on stability and on the maximal absolute or relative performance loss. The motivation for the introduction of suboptimality is that it can lead to significant reductions of online as well as offline computational effort and a reduction of the complexity of the obtained solution. Even though the focus is on quadratic-cost MPC schemes, the presented algorithms can as well be applied to the (simpler) case of piecewise linear cost functions. Another interesting application of the backbone of the algorithm presented can be found in Axehill and Morari (2010). A preliminary version of this work has been published in the conference paper (Axehill, Besselmann, Raimondo, \& Morari, 2011).

\section{Problem statement}

We consider the class of discrete-time mixed logical dynamical (MLD) systems, which is described by the following relations:

$x_{k+1}=A x_{k}+B_{1} u_{k}+B_{2} \delta_{k}+B_{3} z_{k}$,

$E_{2} \delta_{k}+E_{3} z_{k} \leq E_{1} u_{k}+E_{4} x_{k}+e_{5}$,

with the state $x_{k}=\left[\begin{array}{ll}x_{k, c}^{T} & x_{k, b}^{T}\end{array}\right]^{T} \in \mathbb{R}^{n_{x c}} \times\{0,1\}^{n_{x b}}$, and the input $u_{k}=\left[\begin{array}{ll}u_{k, c}^{T} & u_{k, b}^{T}\end{array}\right]^{T} \in \mathbb{R}^{n_{u c}} \times\{0,1\}^{n_{u b}}$ comprising continuous and binary elements. $z_{k} \in \mathbb{R}^{n_{z}}$ and $\delta_{k} \in\{0,1\}^{n_{\delta}}$ denote continuous and binary auxiliary variables, and $A, B_{j}, E_{j}$ and $e_{5}$ denote real matrices and a real vector of appropriate dimensions, respectively. Constraints on the state and the input of an MLD system such as $x_{k} \in \mathcal{X}, u_{k} \in \mathcal{U}$ can be incorporated in (1b). It is assumed that both sets are compact and contain the origin in their interior. An MLD system is called completely well-posed if $\delta_{k}$ and $z_{k}$ are uniquely determined by (1b) for given $x_{k}, u_{k}$, implying that also $x_{k+1}$ is uniquely determined.

Consider the following constrained finite-time optimal control (CFTOC) problem for MLD systems, for a given initial state $x_{k}$ :

$\underset{\boldsymbol{U}_{k}}{\operatorname{minimize}} J\left(\boldsymbol{U}_{k} ; x_{k}\right)$

subject to MLD system dynamics in (1),

$$
x_{k+N} \in \chi_{T} \text {. }
$$

The set $\chi_{T} \subseteq \mathcal{X}, 0 \in \mathcal{X}_{T}$, denotes a compact polyhedral terminal set, constraining the state $x_{k+N}$ at the (finite) final time instance $N$. In quadratic cost optimal control, the cost function is defined as a quadratic function of the states and inputs within the prediction horizon $N$,

$J\left(\boldsymbol{U}_{k} ; x_{k}\right)=V_{T}\left(x_{k+N}\right)+\sum_{i=0}^{N-1} l\left(x_{k+i}, u_{k+i}\right)$

with $V_{T}\left(x_{k+N}\right)=x_{k+N}^{T} P x_{k+N}$, and $l\left(x_{k+i}, u_{k+i}\right)=x_{k+i}^{T} Q x_{k+i}$ $+u_{k+i}^{T} R u_{k+i} . \boldsymbol{U}_{k}=\left\{u_{k}, \ldots, u_{k+N-1}\right\}$ denotes the sequence of control actions, and the weight matrices $Q, P \in \mathbb{S}_{+}^{n_{x}}$ and $R \in \mathbb{S}_{++}^{n_{u}}$ are assumed to be positive (semi)definite.

Explicit MPC for MLD systems requires the parametric solution of the CFTOC problem (2) for a range of different states $x_{k}$, i.e., the solution of a multi-parametric mixed-integer quadratic program (mp-MIQP). In order to mitigate complexity issues within the explicit hybrid MPC framework, we are interested in suboptimal solutions to $\mathrm{mp}$-MIQPs. The aim of this paper is a procedure to compute a suboptimal sequence of control laws $\hat{\boldsymbol{U}}_{k}\left(x_{k}\right)$ to the CFTOC problem (2), such that the resulting suboptimal cost $\hat{J}\left(x_{k}\right)$ satisfies

$\hat{J}\left(x_{k}\right)-J^{*}\left(x_{k}\right) \leq \sigma\left(x_{k}\right)$ for all $x_{k}$ in $\hat{X}_{f} \subseteq \mathcal{X}$, where $J^{*}\left(x_{k}\right)$ denotes the optimal cost, where the finite positive $\sigma\left(x_{k}\right): \mathbb{R}^{n_{x}} \rightarrow \mathbb{R}_{+}$denotes a user-defined suboptimality tolerance, and where $\hat{X}_{f}$ denotes the set of states for which the problem in (2) is feasible. The resulting suboptimal receding horizon control law will be denoted by $\hat{u}_{R H}\left(x_{k}\right)$.

\section{Multi-parametric programming}

In this section, optimization problems that depend on a parameter $\gamma \in \Gamma \subset \mathbb{R}^{n_{\gamma}}$ will be discussed. Throughout the paper, the set $\Gamma$ is assumed to be polyhedral. Both multi-parametric MIQP (mp$\mathrm{MIQP}$ ) problems and multi-parametric QP (mp-QP) problems will be considered in this work. The mp-MIQP problems considered are in the form

$$
\begin{array}{cl}
\underset{x}{\operatorname{minimize}} & \frac{1}{2} x^{T} H x+f^{T} x \\
\text { subject to } & A_{E} x=S_{E} \gamma+b_{E}, \\
& A_{I} x \leq b_{I}, \\
& x_{i} \in\{0,1\} \quad \forall i \in \mathcal{B},
\end{array}
$$

where $\mathscr{B}$ is a set containing the indices to the binary components of $x$. Furthermore, relaxations of the mp-MIQP problem in (4), with some of the relaxed binary variables fixed, are of interest. These problems are in the form

$$
\begin{array}{cl}
\underset{x}{\operatorname{minimize}} & \frac{1}{2} x^{T} H x+f^{T} x \\
\text { subject to } & (4 \mathrm{~b}),(4 \mathrm{c}), \\
& 0 \leq x_{i} \leq 1, \quad \forall i \in \mathscr{B}, \\
& x_{i}=0, \quad \forall i \in \mathscr{B}_{0}, \quad x_{i}=1, \quad \forall i \in \mathscr{B}_{1},
\end{array}
$$

which is an mp-QP problem and $\mathcal{B}_{0} \cup \mathscr{B}_{1} \subseteq \mathscr{B}$. Many contributions have been published in the area of mp-QP and mp-MIQP, see, e.g., Bemporad et al. (2002), Dua and Pistikopoulos (2000) and Dua et al. (2002). The following definitions are fundamental for what follows.

Definition 1. A function $f(x): X \rightarrow \mathbb{R}^{m}$, where $X \subseteq \mathbb{R}^{n}$, is piecewise affine (PWA) if it is possible to partition $X$ into regions $\mathcal{R}_{i}$ such that $f(x)=H^{i} x+k^{i}, x \in \mathcal{R}_{i}$.

Definition 2. A function $f(x): X \rightarrow \mathbb{R}^{m}$, where $X \subseteq \mathbb{R}^{n}$ is a polyhedral set, is polyhedral piecewise affine (PPWA) if it is PWA and the regions $\mathcal{R}_{i}$ are convex polyhedral regions.

Piecewise quadratic functions (PWQ) and polyhedral piecewise quadratic functions (PPWQ) are defined analogously. The most important properties of the $\mathrm{mp}-\mathrm{QP}$ solution are summarized in Theorem 3.

Theorem 3 (Bank et al., 1982). Consider the mp-QP in (5), assume $H \in \mathbb{S}_{+}^{n}$, and $\Gamma$ convex. Then the set of feasible parameters $\Gamma^{*} \subseteq \Gamma$ is convex, the optimizer $x^{*}(\gamma)$ is continuous and PPWA, and the optimal objective function (the value function) $J^{*}(\gamma)$ is continuous, convex and $P P W Q$.

Similarly, the properties of the mp-MIQP solution are summarized as

Theorem 4. Consider the mp-MIQP in (4), assume $H \in \mathbb{S}_{+}^{n}$, and $\Gamma$ convex. Then the set of feasible parameters $\Gamma^{*} \subseteq \Gamma$ is not necessarily convex, the optimizer $x^{*}(\gamma)$ is PWA, but not necessarily continuous, and the optimal objective function $J^{*}(\gamma)$ is PWQ but in general, neither convex nor continuous. An optimal solution to the mp-MIQP has an objective function given by

$J^{*}(\gamma)=\min _{p} J_{p}(\gamma)$, 
where each $J_{p}$ denotes the optimal objective function to an $m p-Q P$, and thus has the properties described in Theorem 3.

Proof. See, e.g., Borrelli (2003). The expression in (6) follows directly from fixing the binary variables in (4) to each possible combination $p$ of binaries, computing the corresponding explicit solutions with value functions $J_{p}(\gamma)$, and finally taking $J^{*}(\gamma)$ as the point-wise minimizer.

\section{Suboptimal branch and bound}

Branch and bound is a method that in many cases can solve an MIQP problem more efficiently than explicitly enumerating all possible combinations of integer variables by organizing the search space in the form of a binary search tree. For more information, see, e.g., Wolsey (1998). In this section, it is investigated how branch and bound can be used to compute suboptimal solutions to nonparametric MIQP-problems.

Definition 5. A $\sigma$-suboptimal solution is a solution to an optimization problem with a corresponding objective function value $\hat{J}$ that satisfies $\hat{J}-J^{*} \leq \sigma$ if $J^{*}<\infty$.

A formal algorithmic description of a branch and bound algorithm that computes an $\left(\epsilon+(1+\rho) \xi+\rho J^{*}\right)$-suboptimal solution to an MIQP optimization problem $P$ is presented in Algorithm 1, where $P$ is an MIQP problem with feasible set $\delta, P_{i}$ is an MIQP problem with feasible set $\delta_{i}$ constructed from $P$ but additionally subject to a set of constraints locking a subset of the binary variables either to 0 or to 1 , and $\underline{P}_{i}$ is the QP relaxation of $P_{i}$ with feasible set $\underline{s}_{i}$. Furthermore, $\hat{x}$ and $\hat{J}$ are the solution and objective function value, respectively, of the best known integer feasible solution so far, $x_{i}$ and $J_{i}$ are the $\xi$-suboptimal solution and objective function value to rellaxation $\underline{P}_{i}$, and LIST is a sorted list implementing a priority queue. The ordering of the nodes (also called vertices) in LIST corresponds to the choice of the tree exploration strategy (e.g., depth first). The constant $\epsilon \geq 0$ is an allowance parameter that controls the absolute suboptimality of the solution and the constant $\rho \geq 0$ is a relative allowance parameter that controls the relative suboptimality of the solution. The constant $\xi \geq 0$ bounds the suboptimality of the solutions to the relaxations $\underline{P}_{i}$. In the optimal case it holds that $\epsilon=\rho=\xi=0$. Even though $\epsilon, \rho$ and $\xi$ affect the suboptimality of the computed solution in a similar way, their origins are different. Typically, $\epsilon$ and $\rho$ are intentionally chosen greater than zero by the user in order to try to reduce the size of the tree for the price of suboptimality, while $\xi$ is often a result of an approximation scheme used to compute solutions to the relaxations. The effect of a non-zero $\epsilon$ is studied in more detail in Ibaraki, Muro, Murakami, and Hasegawa (1983). The effect of a non-zero $\xi$ appears to be less investigated in the literature. On termination, a solution $\hat{x}$ is returned by Algorithm 1 with a corresponding objective function value $\hat{J}$ and a quality guaranteed by Theorem 6 .

Theorem 6. Assume $\epsilon, \rho, \xi \in \mathbb{R}_{+}, J^{*} \geq 0$ and that $\xi$-suboptimal solutions can be computed to the relaxations $\underline{P}_{i}$. Furthermore, assume that $P$ is feasible. Then, when Algorithm 1 terminates it holds that $\hat{x}$ is an $\left(\epsilon+(1+\rho) \xi+\rho J^{*}\right)$-suboptimal solution to $P$.

Proof. Denote with $B$ the binary tree explored by Algorithm 1 and with $V(B)$ its vertex set. Each vertex in $V(B)$ represents a problem denoted $P_{i}$ in Algorithm 1. Denote the root vertex $r \in V(B)$ and a leaf containing an optimal solution to $P$ by $\ell \in V(B)$. Define $J(v)$ as the optimal objective function value to the relaxation at vertex $v$ denoted $P(v)$. Furthermore, define an approximate solution to $\underline{P}(v)$ as $\underset{\sim}{x}(v)$ with objective function value $J(v)$ that satisfies $J(v) \leq$ $J(v) \leq J(v)+\xi$. Finally, let $\hat{J}(v)$ denote the objective function

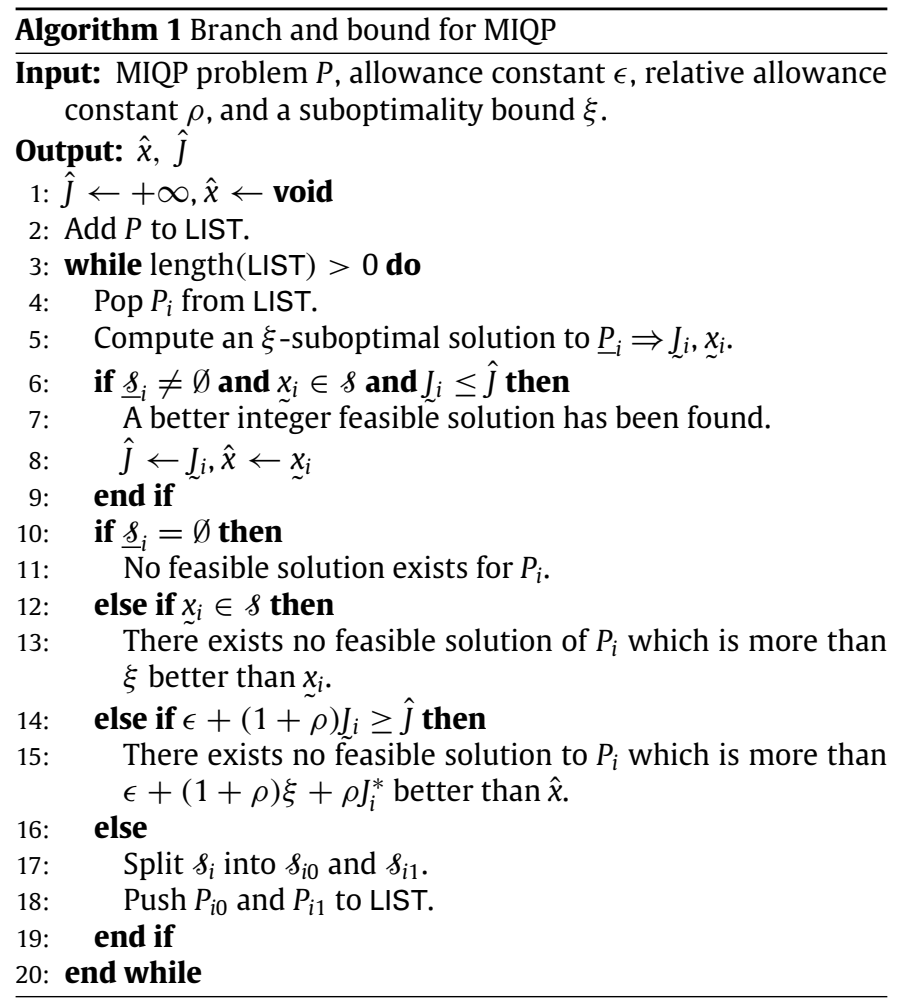

value of the incumbent at vertex $v$ before any update of it has been performed. Consider the unique $r, \ell$-trail $T(\ell) \subseteq B$ of length $n_{b}$. Along this trail, it holds by the construction of the tree that $J(v) \leq J^{*}, \forall v \in T(\ell)$. In particular it holds that $J(\ell)=J^{*}$. If Allgorithm 1 does not explore all vertices $v \in T(\ell)$, it then either holds that $\underset{\sim}{\underset{\sim}{x}}(v) \in \delta$ (cut by line 12) or that $\epsilon+(1+\rho) J(v) \geq \hat{J}(v)$ at a vertex $\tilde{v} \in T(\ell)$ (cut by line 14, or line 10 since infeasibility is represented by infinite cost). First, if $x(v) \in \delta$, then it holds that $\underset{\sim}{J}(v) \leq \underline{J}(v)+\xi \leq J^{*}+\xi$. Hence, after the update of the incumbent (lines 6-9), it holds that $\hat{J}\left(v^{+}\right)-J^{*} \leq \xi$, where $v^{+}$is the vertex explored after $v$. Second, if $\epsilon+(1+\rho) J(v) \geq \hat{J}(v)$, it holds that $\hat{J}(v) \leq \epsilon+(1+\rho) \xi+(1+\rho) J(v) \Rightarrow \hat{J}(v)-J^{*} \leq$ $\epsilon+(1+\rho) \xi+\rho J^{*} \Rightarrow \hat{J}\left(v^{+}\right)-J^{*} \leq \epsilon+(1+\rho) \xi+\rho J^{*}$. Hence, $\tilde{x}\left(v^{+}\right)$has been proven to be $\left(\epsilon+(1+\rho) \xi+\rho J^{*}\right)$-suboptimal. Since the incumbent can only be improved during the execution of Algorithm 1 (follows from line 6), the desired result follows.

\section{5. mp-MIQP using branch and bound}

In this section, an algorithm for computing the parametric solution to mp-MIQP problems using multi-parametric branch and bound (mp-BnB) will be presented. It extends ideas presented in Acevedo and Pistikopoulos (1997) for multi-parametric mixed-integer linear programs, to multi-parametric mixed-integer quadratic programs. Furthermore, compared to Acevedo and Pistikopoulos (1997) where only the optimal case is considered, this work considers a more general case where the user can intentionally relax the optimality requirement and specify absolute and relative suboptimality bounds a priori. Moreover, in this work the case when the mp-QP relaxations in the nodes are not solved to optimality is considered, which is motivated by the recent increased interest in approximate mp-QP (i.e., approximate explicit linear MPC).

Definition 7. A $\sigma(\gamma)$-suboptimal solution, with $\sigma(\gamma): \mathbb{R}^{n_{\gamma}} \rightarrow \mathbb{R}_{+}$ finite and non-negative, is a solution to a parametric optimization 


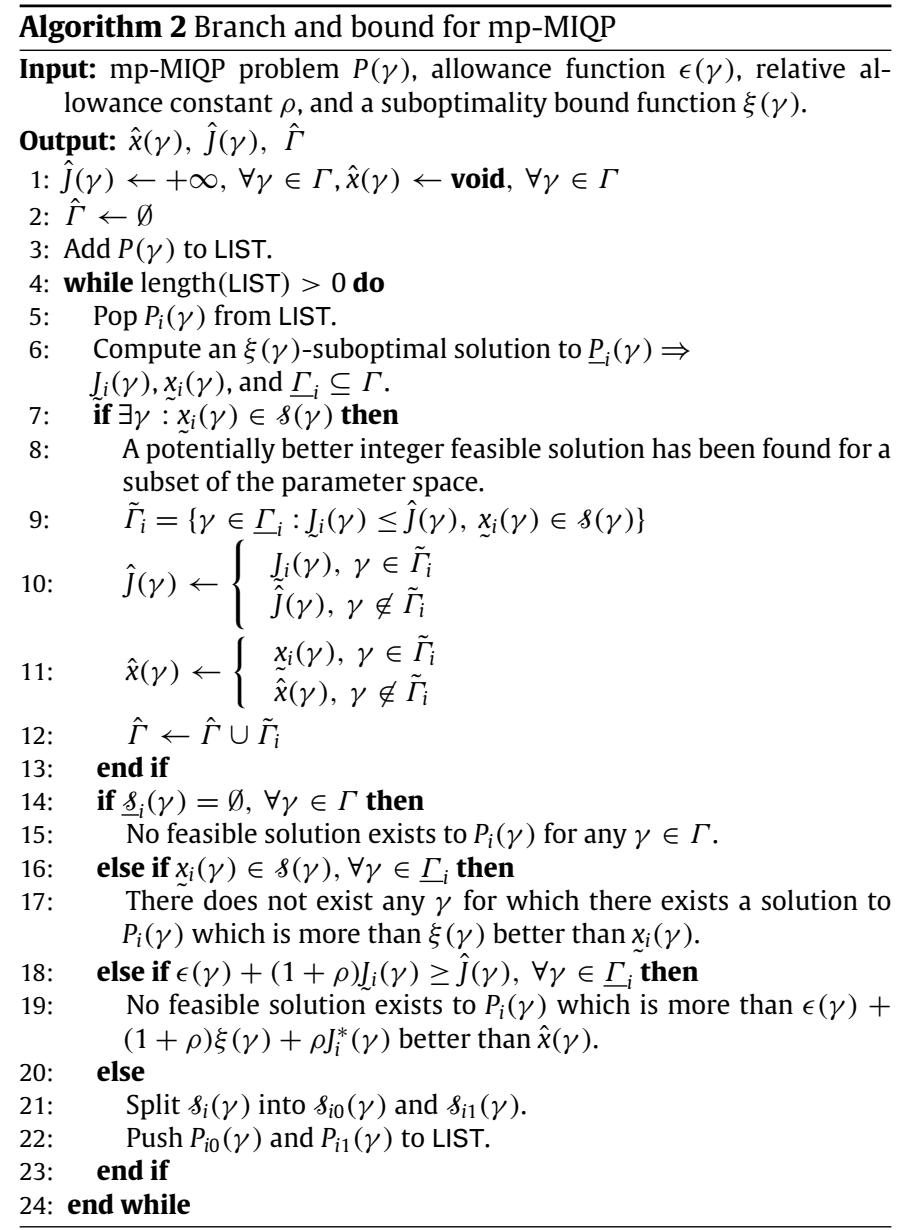

problem with parameter $\gamma$ with a corresponding value function $\hat{J}(\gamma)$ for which it holds that $\hat{J}(\gamma)-J^{*}(\gamma) \leq \sigma(\gamma), \forall \gamma \in \Gamma^{*}$, where $\Gamma^{*}$ is the set of parameters for which there exists a feasible solution.

In this work the mp-MIQP problem is solved using the branch and bound method, where mp-QP problems (relaxations) are solved in the nodes. This approach provides two benefits compared to explicit enumeration of all feasible binary sequences ("switching sequences"). First, there is a potential of handling larger problems with more binary variables, with the same motivation as for non-parametric branch and bound. Second, it provides an efficient framework for computation of suboptimal solutions. By relaxing the optimality requirement, many integer feasible solutions are possible to cut away during the branch and bound process, since they only marginally contribute to the optimal solution. In the parametric case, this means that the number of $\mathrm{mp}-\mathrm{QP}$ problems to be solved offline typically can be significantly reduced. By introducing the possibility to use approximate mp-QP solutions for the relaxations in the nodes, the cost for these can be reduced, the comparison of the different bounds can potentially become computationally cheaper, and the final explicit solution might be simplified even further.

The $\mathrm{mp}-\mathrm{BnB}$ is presented in detail in Algorithm 2, where the value functions are assumed to be infinite for those $\gamma$ where the problem is infeasible.

The used variables are defined analogously as in Algorithm 1 apart from a possible parameter dependence. The sets $\hat{\Gamma}$ and $\underline{\Gamma}_{i}$ denote the obtained set of feasible parameters for the best known integer feasible solution so far and the obtained set of feasible parameters for the relaxation in node $i$, respectively. The user-defined allowance function $\epsilon(\gamma) \geq 0$ is a function that controls the absolute suboptimality of the solution. Similarly, the constant $\rho \geq 0$ is a relative allowance constant that controls the relative suboptimality of the solution. The function $\xi(\gamma) \geq 0$ should be chosen as a bound on the suboptimality of the mp-QP solutions to the relaxations. If a globally optimal parametric solution is sought for, then $\epsilon(\gamma) \equiv 0, \rho=0, \xi(\gamma) \equiv 0, \forall \gamma$. When Algorithm 2 terminates, the solution has the quality stated in Theorem 8 .

Theorem 8. Assume that $0 \leq \epsilon(\gamma)<\infty, 0 \leq \xi(\gamma)<\infty, \forall \gamma \in$ $\Gamma, 0 \leq \rho<\infty$, and that $\xi(\gamma)$-suboptimal solutions to $\underline{P}_{i}(\gamma)$ can be computed. Then, when Algorithm 2 terminates it holds that $\hat{x}(\gamma)$ is $\left(\epsilon(\gamma)+(1+\rho) \xi(\gamma)+\rho J^{*}(\gamma)\right)$-suboptimal.

Proof. Can be performed analogously to the proof of Theorem 6 while considering the parametric cut conditions used in Algorithm 2. $\square$

Compared to Algorithm 1, Algorithm 2 involves some significantly more complicated operations. On line 6 , this algorithm solves relaxations in the form in (5) parametrically instead of only for a single $\gamma$. On line 9 and line 18, two PWQ functions are compared. An algorithm that solves the relatively advanced task to compare PWQ functions is presented in Section 6.

This section is concluded with a corollary describing the set of feasible parameters $\hat{\Gamma}$ of the parametric solution returned by Algorithm 2.

Corollary 9. Denote the set of feasible parameters for a globally optimal solution $\Gamma^{*} \subseteq \Gamma$. Assume the assumptions in Theorem 8 hold. Then $\hat{\Gamma}=\Gamma^{*}$.

Proof. $\hat{\Gamma} \supseteq \Gamma^{*}$ follows from Theorem 8. $\hat{\Gamma} \subseteq \Gamma^{*}$ follows from the fact that the feasible set of parameters cannot be larger for a suboptimal solution than for an optimal solution.

\section{Comparison of PWQ functions}

In this section an algorithm to compare PWQ functions is presented, Besselmann (2010). This is the key to extending the result in Acevedo and Pistikopoulos (1997) to the more complicated quadratic cost case. Note that also other comparison strategies are possible, e.g., the one in Alessio and Bemporad (2006) which is based on DC programming, Horst and Thoai (1999).

The best-so-far solution $\hat{J}(\gamma)$ in Algorithm 2 is PWQ but not necessarily PPWQ making it difficult to store, to evaluate and to handle. However, $\hat{J}(\gamma)$ is composed of PPWQ integer feasible solutions $J_{p}(\gamma), p=1, \ldots, n_{p}$. Instead of working with $\hat{J}(\gamma)$ directly, one can thus substitute $\hat{J}(\gamma)$ by $\min _{p} J_{p}(\gamma)$. The following corollary ensures that the comparisons in lines 10 and 19 of Algorithm 2 consequently boil down to comparisons of PPWQ functions.

Corollary 10. Let $\hat{J}(\gamma): \hat{\Gamma} \rightarrow \mathbb{R}$ be the best-so-far solution from Algorithm 2 and let $J(\gamma): \Gamma \rightarrow \mathbb{R}$ be an arbitrary function with $\Gamma, \hat{\Gamma} \subseteq \mathbb{R}^{n_{\gamma}}$. For a given $\gamma \in(\hat{\Gamma} \cap \Gamma)$ it holds $J(\gamma) \leq \hat{J}(\gamma) \Leftrightarrow$ $J(\gamma) \leq J_{\sim}(\gamma) \forall p$, and $J(\gamma) \geq \hat{J}(\gamma) \Leftrightarrow \exists p: J(\gamma) \geq J_{\sim}(\gamma)$.

Definition 11. We call a region $\mathcal{R} \subset \mathbb{R}^{n_{x}}$ dominated by a finite set of regions $\left\{\mathcal{R}_{i}\right\}_{i=1}^{n} \subset \mathbb{R}^{n_{x}}$, iff $\forall \gamma \in \mathcal{R}, \exists i \in\{1, \ldots, n\}: \gamma \in$ $\mathcal{R}_{i} \wedge J_{i}(\gamma) \leq J(\gamma)$. A dominated region is also called redundant.

In order to save computation time and storage space, regions are treated as atomic units, i.e., regions are not split into subregions, only entire regions are marked as redundant and all comparisons are carried out region-by-region. A region-by-region version of the conditions in Corollary 10 is 
Corollary 12. Let $\hat{J}(\gamma): \hat{\Gamma} \rightarrow \mathbb{R}$ be the best-so-far solution from Algorithm 2 and let $J(\gamma): \Gamma \rightarrow \mathbb{R}$ be an arbitrary function with $\Gamma, \hat{\Gamma} \subseteq \mathbb{R}^{n_{\gamma}}$. For a given region $\mathcal{R} \subseteq(\hat{\Gamma} \cap \Gamma)$ it holds that $\exists \gamma \in \mathcal{R}$ : $J(\gamma) \leq \hat{J}(\gamma) \Leftrightarrow \exists \gamma \in \mathcal{R}: J(\gamma) \leq \bar{J}_{p}(\gamma) \forall p$, and $J(\gamma) \geq \hat{J}(\gamma) \forall \gamma \in$ $\mathcal{R} \Leftrightarrow \forall \gamma \in \mathcal{R}, \exists p: J(\gamma) \geq J_{p}(\gamma)$.

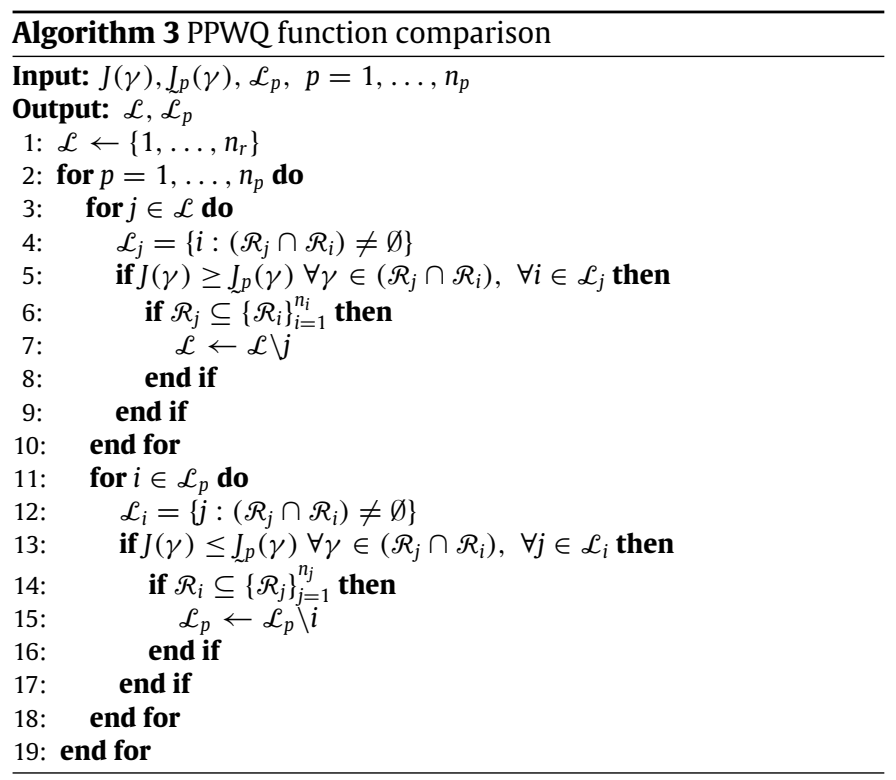

Based on Corollary 12, the comparison of PWQ functions can be executed as outlined in Algorithm 3. The algorithm takes a PPWQ function $J(\gamma)$ and compares it to a set of PPWQ functions $J_{p}(\gamma)$, partition-by-partition and region-by-region. The lists $\mathcal{L}$ and $\mathcal{L}_{p}$ indicate non-redundant regions, while the lists $\mathcal{L}_{i}$ and $\mathcal{L}_{j}$ indicate which regions intersect with each other. Algorithm 3 can be used in line 10 as well as in line 19 of Algorithm 2. After line 10, the best-so-far solution can be updated by simply appending $J(\gamma)$ to $J_{p}(\gamma)$, and $\mathcal{L}$ to $\mathcal{L}_{p}$. In line 19 , we do not want to update the bestso-far solution, thus it is sufficient to compute $\mathcal{L}$ without updating $\mathcal{L}_{p}$, and lines $11-18$ of Algorithm 3 do not have to be executed. If $\mathcal{L}=\emptyset$ after the execution of Algorithm 3, the condition in line 19 of Algorithm 2 is fulfilled.

The proposed procedure is only identifying regions which are dominated by a single partition. The conditions of redundancy in Corollary 12 are thus strengthened to $\exists \gamma \in \mathcal{R}: J(\gamma) \leq J_{p}(\gamma) \forall p$ $\Rightarrow \forall p \exists \gamma \in \mathcal{R}: J(\gamma) \leq J_{p}(\gamma)$, and $\forall \gamma \in \mathcal{R}, \exists p: J(\gamma) \geq$ $J_{p}(\gamma) \Leftarrow \exists p: J(\gamma) \geq J_{\sim}(\gamma) \forall \gamma \in \mathcal{R}$. In fact $J(\gamma)$ may also contain redundant regions which are not dominated by a single partition, but by a set of partitions. Identifying also these regions is conceptually straightforward, but the computational effort would grow significantly.

At the core of Algorithm 3, redundant regions are determined by pairwise comparisons of quadratic cost functions. This is equivalent to checking if the difference of two quadratic functions, and thus its minimum, is non-negative on the common domain. We are proposing to compare the quadratic cost functions by solving the quadratic program

$\min _{\gamma}\left\{J_{1}(\gamma)-J_{2}(\gamma) \mid \gamma \in\left(\mathcal{R}_{1} \cap \mathcal{R}_{2}\right)\right\}$.

Note that the difference of two quadratic functions is not necessarily convex. If the difference function is convex, the quadratic program (7) can be solved by a standard QP-solver. If the difference function is non-convex, the optimization problem is a non-convex quadratic program, which is known to be $\mathcal{N} \mathcal{P}$-hard, Pardalos and Vavasis (1991). In this situation, a spatial branch and bound algorithm is employed built on the ideas in McCormick (1976), for which an implementation is available in YALMIP, Löfberg (2004). Since the optimization problem in (7) does not have to be solved to optimality, the minimization can be terminated if one of the stopping criteria, i.e., a lower bound greater than zero or an upper bound less than zero, is met.

\section{Suboptimal hybrid MPC scheme}

Explicit MPC for MLD systems requires the solution of an mpMIQP problem, which we now know can be efficiently solved optimally and suboptimally using Algorithm 2. Given this new framework, it is interesting to discuss different choices of the suboptimality tolerance $\sigma\left(x_{k}\right)=\epsilon\left(x_{k}\right)+(1+\rho) \xi\left(x_{k}\right)+\rho J^{*}\left(x_{k}\right)$.

I. Optimal solution: The optimal solution to the CFTOC problem (2) is obtained by selecting $\sigma\left(x_{k}\right) \equiv 0$.

II. Absolute performance bound: In general the absolute performance bound $\epsilon\left(x_{k}\right)$ can take on the form of any polyhedral piecewise quadratic function. By selecting a large enough absolute suboptimality tolerance, the algorithm terminates after computing any solution feasible for all $x_{k} \in \hat{X}_{f}$.

III. Relative performance bound: The user can also specify a performance bound relative to the optimal cost function $\sigma\left(x_{k}\right)=$ $\rho J^{*}\left(x_{k}\right)$ with $\rho \in \mathbb{R}_{+}$.

IV. Suboptimal solutions to relaxations: If the relaxations are computed $\xi(\gamma)$-suboptimally, the returned solution in general becomes suboptimal. See Theorem 8 for more details.

V. Stability: Depending on the choice of the suboptimality tolerance, closed-loop stability can also be guaranteed.

In order to guarantee stability, the set $\mathcal{X}_{T}$ and the terminal penalty $V_{T}$ are required to satisfy the following assumption.

Assumption 13. The design parameters $V_{T}$ and $x_{T}$ in (2) are such that, given an auxiliary control law $\kappa_{T}(x)$, a $\mathcal{K}$-function $\alpha_{2}(|x|)$, and a neighborhood of the origin $\mathcal{N} \subseteq \mathcal{X}_{T}$

- $\kappa_{T}(x) \in U, \forall x \in X_{T}$

- $\mathcal{X}_{T}$ is invariant for system (1) in closed loop with $\kappa_{T}(x)$

- $V_{T}(x) \leq \alpha_{2}(x), \forall x \in \mathcal{N}$

- $V_{T}\left(f\left(x, \kappa_{T}(x)\right)\right)-V_{T}(x) \leq-l\left(x, \kappa_{T}(x)\right), \forall x \in X_{T}$

Assumption 13 is a standard stabilization condition for hybrid MPC, see Lazar and Heemels (2009). We now recall Theorem 16 in Lazar and Heemels (2009) whose assumptions hold for the suboptimal MPC scheme here proposed (for the meaning of epsilon-asymptotic stability (epsilon-AS) see Definition 3 in Lazar and Heemels (2009)).

Theorem 14 (Lazar \& Heemels, 2009). Under Assumption 13, given a suboptimality tolerance $\sigma(x)=\sigma$, the origin of system (1) in closed loop with $\hat{u}_{R H}(x)$ is $\varepsilon$-AS in $\hat{X}_{f}$. Moreover, if the suboptimality tolerance is state dependent, and there exists a $\mathcal{K}$-function $\alpha_{3}$ such that $-\alpha_{1}(|x|)+\sigma(x) \leq-\alpha_{3}(|x|)$ for all $x \in \hat{\chi}_{f}$, then the origin of system (1) in closed loop with $\hat{u}_{R H}(x)$ is asymptotically stable in $\hat{X}_{f}$.

\section{Numerical examples}

In this section Algorithm 2 is tested in numerical experiments using Matlab 7.10 on a computer with four processors of type Intel Xeon E5540 with 24 GB RAM running Debian 5.0.6. The CPLEX version used was 11.1, the MPT version 2.6.2, and the YALMIP version was 3 . In the example, the algorithm was applied to 9 stable random hybrid MPC problems of the type in (2) with 4 real-valued states, 2 real-valued control signals, 3 binary-valued control signals, and no auxiliary variables. The real-valued inputs were constrained by random upper and lower bounds, whereas no state 

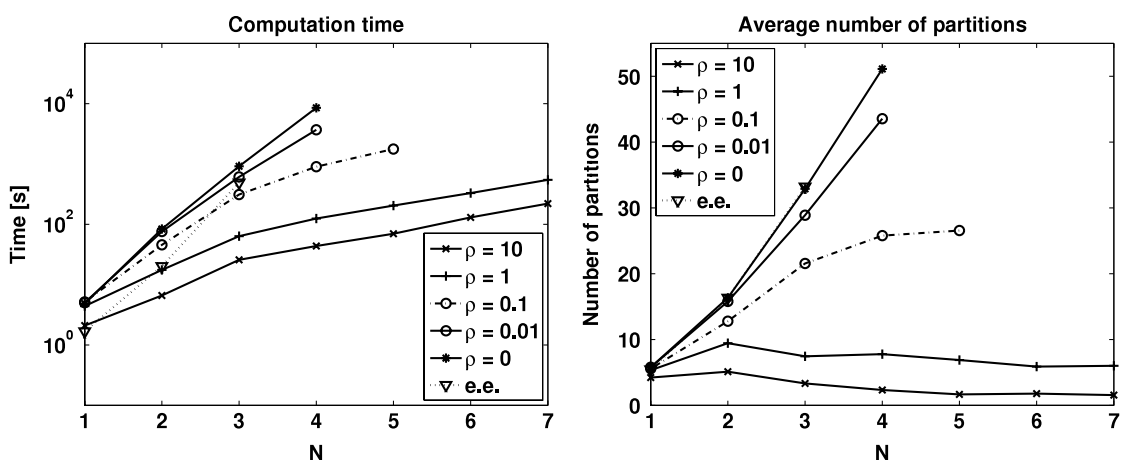

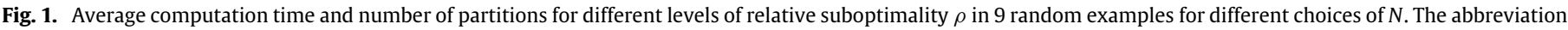
e.e. denotes explicit enumeration.

constraints were enforced. The prediction horizon length $N$ was varied in the range $1-7$ steps, resulting in $2^{3 \cdot N}$ possible switching sequences. The matrices $Q$ and $R$ were chosen as a random positive semidefinite and a random positive definite matrix, respectively, $P$ was chosen equal to $Q$, and a relative performance bound $\rho$ was used as described as Option III in Section 7. The problem was solved parametrically for all $\left\|x_{k}\right\|_{\infty} \leq 1$. Explicit enumeration is also shown as a comparison. The average computation time and the average number of partitions from the 9 examples for each prediction horizon length are shown in Fig. 1. All computation times presented include removal of redundant regions using the algorithm presented in Section 6. The traditional explicit enumeration approach was not able to finish the 9 examples for $N=4$ within the time of the experiments. Furthermore, it can also be seen that if Algorithm 2 is run with the aim for an optimal solution $(\rho=0)$, the number of partitions is typically the same as from explicit enumeration after overlap reduction.

The conclusions drawn from the experiment are that, firstly, Algorithm 2 outperforms traditional explicit enumeration in terms of computation time for computing an optimal solution to problems already with modest complexity and, secondly, the introduction of suboptimality reduces the computation times even further as well as the complexity (number of partitions) of the resulting explicit solution. This effect becomes more pronounced for longer prediction horizons, with a large number of possible switching sequences. It also follows from the result of the experiment that this reduction continues as the level of suboptimality is increased and the maximum reasonable level is eventually given by what performance of the closed-loop system that is acceptable in a particular application.

\section{References}

Acevedo, J., \& Pistikopoulos, E. N. (1997). A multiparametric programming approach for linear process engineering problems under uncertainty. Industrial $\mathcal{E}$ Engineering Chemistry Research, 36(3), 717-728.

Alessio, A., \& Bemporad, A. (2006). Feasible mode enumeration and cost comparison for explicit quadratic model predictive control of hybrid systems. In Preprints of the 2nd Conf. on Analysis and Design of Hybrid Systems. Alghero, Italy.

Axehill, D., Besselmann, T., Raimondo, D. M., \& Morari, M. (2011). Suboptimal explicit hybrid MPC via branch and bound. In Proceedings of the 18th IFAC world congress (pp. 10281-10286). Milan, Italy.

Axehill, D., \& Morari, M. (2010). Improved complexity analysis of branch and bound for hybrid MPC. In Proceedings of the 49th IEEE conference on decision and control, (pp. 4216-4222). Atlanta, USA.

Bank, B., Guddat, J., Klatte, D., Kummer, B., \& Tammer, K. (1982). Non-linear parametric optimization. Berlin: Akademie-Verlag.

Baotic, M. (2005). Optimal Control of Piecewise Affine Systems - A Multi-Parametric Approach. PhD thesis. Automatic Control Laboratory, ETH Zurich.

Bemporad, A., Morari, M., Dua, V., \& Pistikopoulos, E. N. (2002). The explicit linear quadratic regulator for constrained systems. Automatica, 38(1), 3-20.

Besselmann, T. (2010). Constrained Optimal Control - Piecewise Affine and Linear Parameter-Varying Systems. PhD thesis. Automatic Control Laboratory, ETH Zurich.
Borrelli, F. (2003). Lecture notes in control and information sciences: Vol. 290. Constrained optimal control of linear and hybrid systems. Springer-Verlag.

Camacho, E. F., \& Bordons, C. (2004). Model predictive control. Springer.

Dua, V., Bozinis, N. A., \& Pistikopoulos, E. N. (2002). A multiparametric programming approach for mixed-integer quadratic engineering problems. Computers $\mathcal{E}$ Chemical Engineering, 26, 715-733.

Dua, V., \& Pistikopoulos, E. N. (2000). An algorithm for the solution of multiparametric mixed integer linear programming problems. Annals of Operations Research, 99, 123-139.

Heemels, W. P. M. H., de Schutter, B., \& Bemporad, A. (2001). Equivalence of hybrid dynamical models. Automatica, 37(7), 1085-1091.

Horst, R., \& Thoai, N. V. (1999). DC programming: overview. Journal of Optimization Theory and Applications, 103, 1-43.

Ibaraki, T., Muro, S., Murakami, T., \& Hasegawa, T. (1983). Using Branch-and-Bound Algorithms to obtain suboptimal solutions. Zeitschrift für Operations Research, 27, 177-202.

Kvasnica, M., Grieder, P., Baotic, M., \& Morari, M. (2004). Multi-Parametric Toolbox (MPT). In Proceedings of the international workshop on hybrid systems: computation and control (pp. 448-462). Philadelphia, USA.

Lazar, M., \& Heemels, W. (2009). Predictive control of hybrid systems: input-tostate stability results for sub-optimal solutions. Automatica, 45(1), 180-185.

Löfberg, J. (2004). YALMIP : A Toolbox for Modelling and Optimization in MATLAB. In CCA/ISIC/CACSD. Taipei, Taiwan.

McCormick, G. P. (1976). Computability of global solutions to factorable nonconvex programs: part I - convex underestimating problems. Mathematical Programming, 10, 147-175.

Pardalos, P. M., \& Vavasis, S. A. (1991). Quadratic programming with one negative eigenvalue is NP-hard. Journal of Global Optimization, 1(1), 15-22.

Summers, S., Jones, C. N., Lygeros, J., \& Morari, M. (2011). A multiresolution approximation method for fast explicit model predictive control. IEEE Transactions on Automatic Control, 56(11), 2530-2541.

Wolsey, L. A. (1998). Integer programming. John Wiley \& Sons, Inc.

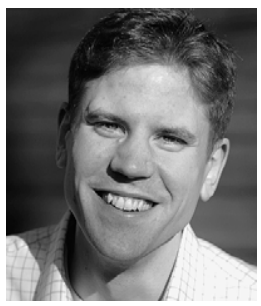

Daniel Axehill was born in Tranås, Sweden, in 1978. He received his M.Sc. degree in Applied Physics and Electrical Engineering in 2003. Furthermore, he received the degree of Lic.Eng. in Automatic Control in 2005 and the Ph.D. degree in Automatic Control in 2008. All three degrees are from Linköping University, Linköping, in Sweden. In year 2006 he spent three months at UCLA in Los Angeles. From January 2009 and until November 2010 he worked as a post-doc at the Automatic Control Laboratory at ETH Zurich. He is currently employed as an Assistant Professor at the Division of Automatic Control at Linköping University. His research interests are related to optimization, optimal control, hybrid systems, signal processing, and applications of control.

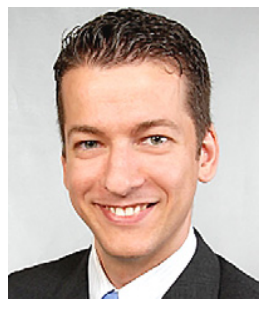

Thomas Besselmann was born 1980 in Thuine, Germany. In 2003 he received his B.Sc. degree in General Engineering Science, and in 2005 his diploma degree in Mechatronics from Hamburg University of Technology in Hamburg, Germany. In 2010 he received his Ph.D. degree at the Automatic Control Laboratory, ETH Zurich, Switzerland. Currently he is employed as a Scientist in the Control \& Optimization group at ABB Corporate Research, Baden-Dättwil, Switzerland. His research interests include high-speed control methods for constrained systems, in particular model predictive control, and their application to automotive and power electronics systems. 


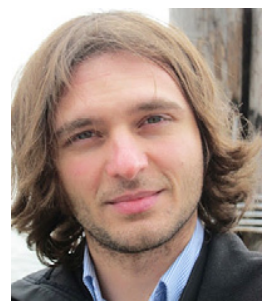

Davide Martino Raimondo was born in Pavia, Italy, in 1981. He received the B.Sc. and M.Sc. in Computer Engineering, and the Ph.D. in Electronic, Computer Science and Electric Engineering from the University of Pavia, Italy, in 2003, 2005, and 2009, respectively. As a Ph.D. student he held a visiting position at the Department of Automation and Systems Engineering, University of Seville, Spain. From January 2009 to December 2010 he was a postdoctoral fellow in the Automatic Control Laboratory, ETH Zürich, Switzerland. From March 2012 to June 2012 and from August 2013 to September 2013 he was a visiting scholar in Prof. Braatz's Group, Department of Chemical Engineering, MIT, USA. Since December 2010 he has been an Assistant Professor at University of Pavia, Italy. He is the author or coauthor of more than 50 papers published in refereed journals, edited books, and refereed conference proceedings. His current research interests include optimization-based control, fault-tolerant control, autonomous surveillance and control of glycemia in diabetic patients.

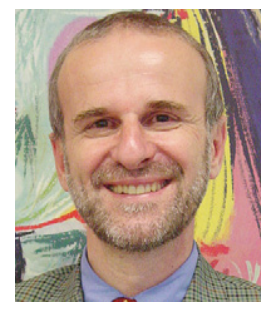

Manfred Morari was head of the Department of Information Technology and Electrical Engineering at ETH Zurich from 2009 to January 2012.

He was head of the Automatic Control Laboratory from 1994 to 2008. Before that he was the McCollum-Corcoran Professor of Chemical Engineering and Executive Office for Control and Dynamical Systems at the California Institute of Technology. He obtained the diploma from ETH Zurich and the Ph.D. from the University of Minnesota both in chemical engineering. His interests are in hybrid systems and the control of biomedical systems. In recognition of his research contributions he received numerous awards, among them the Donald P. Eckman Award, the John R. Ragazzini Award and the Richard E. Bellman Control Heritage Award of the American Automatic Control Council, the Allan P. Colburn Award and the Professional Progress Award of the AIChE, the Curtis W. McGraw Research Award of the ASEE, Doctor Honoris Causa from Babes-Bolyai University, Fellow of IEEE, IFAC and AIChE, the IEEE Control Systems Technical Field Award, and was elected to the National Academy of Engineering (US). Manfred Morari has held appointments with Exxon and ICI plc and serves on the technical advisory boards of several major corporations. 